Int. J. Dev. Biol. 61: 35-42 (2017)

doi: $10.1387 / \mathrm{ijdb} .160160 \mathrm{yy}$

\title{
YelA, a putative Dictyostelium translational regulator, acts as antagonist of DIF-1 signaling to control cell-type proportioning
}

\author{
YOKO YAMADA*, CHRIS SUGDEN and JEFFREY G. WILLIAMS \\ School of Life Sciences, University of Dundee, Dundee, UK
}

\begin{abstract}
DIF-1 (differentiation-inducing factor1) is a polyketide produced by Dictyostelium prespore cells which induces initially uncommitted cells to differentiate as prestalk cells. Exposure of cells to DIF-1 causes transitory hypo-phosphorylation of seven serine residues in YelA, a protein with a region of strong homology to the MIF4G domain of the eukaryotic initiation factor elF4G. Based upon its domain architecture, which in one important aspect closely resembles that of Death-Associated Protein 5 (DAP5), we predict a role in stimulating internal ribosome entry-driven mRNA translation. The two paradigmatic DIF-1 inducible genes are ecmA (extracellular matrixA) and ecmB. In support of a YelA function in DIF-1 signaling, a YelA null strain showed greatly increased expression of ecmA and ecmB in response to DIF-1. Also, during normal development in the null strain, expression of the two genes is accelerated. This is particularly evident for ecm $B$, a marker of stalk tube and supporting structure differentiation. Mutants in DIF-1 bio-synthesis or signaling display a rudimentary or no basal disc and, conversely, YelA null mutants produce fruiting bodies with a highly enlarged basal disc that ectopically expresses a stalk tube-specific marker. Thus YelA acts as an antagonist of DIF-1 signaling, with a consequent effect on cell type proportioning and it is predicted to act as a translational regulator.
\end{abstract}

KEY WORDS: Dictyostelium, DIF-1, MIF4G domain, translational regulation, DAP5

\section{Introduction}

Dictyostelium discoideum is an amoebozoan that undergoes a remarkable change, from unicellular growth to multicellular development, when its food source is depleted. The end point of development is a fruiting body, composed of a ball of spores supported by a cellular stalk that is embedded into a basal disc which is also composed of dead, vacuolated stalk cells. These two terminal stalk cell types derive from different precursor prestalk sub-types: pstA, pstAB, pstO, pstU and pstB cells (Gaudet et al., 2008; Yamada et al., 2010); and see Fig. 5A). Differentiation into prestalk and prespore cells is regulated by extracellular signaling molecules; cAMP induces prespore differentiation (Wang et al., 1988) and DIF-1, a polyketide, acts antagonistically to cAMP to induce prestalk differentiation (Kay et al., 1999). The cells that respond to DIF-1 by becoming prestalk cells appear to be a preenriched population: exposed to starvation at a particular phase of the cell cycle and hyper-sensitive to DIF-1 (Thompson et al., 2000).

DIF-1 signaling is normally assayed in monolayer cells developing under buffer in a petri dish. Under such conditions it is not possible to decide which kind of prestalk cells are induced. However, whole organism analysis of mutants deficient in DIF-1 production or DIF-1 signaling suggest that pstB and pstO differentiation are affected but to different degrees; PstB cells form the basal disc while pstO cells form the upper part of the stalk (Fig. 5A). Basal disc tissue itself is greatly reduced in DIF-1 mutants but the pstO region is maintained, albeit with a different pattern of gene expression (Keller et al., 2008). The defect in pstO cells derives from an analysis of ecmA and $e c m B$, the markers that originally defined the cell types and that encode two closely related proteins of the extracellular matrix (Williams et al., 1987; McRobbie et al., 1988; Jermyn et al., 1989).

At least two discrete transcriptional signaling pathways mediate prestalk gene expression (Yamada etal., 2013), and these are best

Abbreviations used in this paper: DAP, death-associated protein; DIF, differentiationinducing factor; ECM, extracellular matrix; YelA, yellow mounds A.

\footnotetext{
*Address correspondence to: Yoko Yamada. School of Life Sciences, University of Dundee, Dow St., Dundee, DD1 5EH, UK. e-mail: y.yamada@dundee.ac.uk
} 


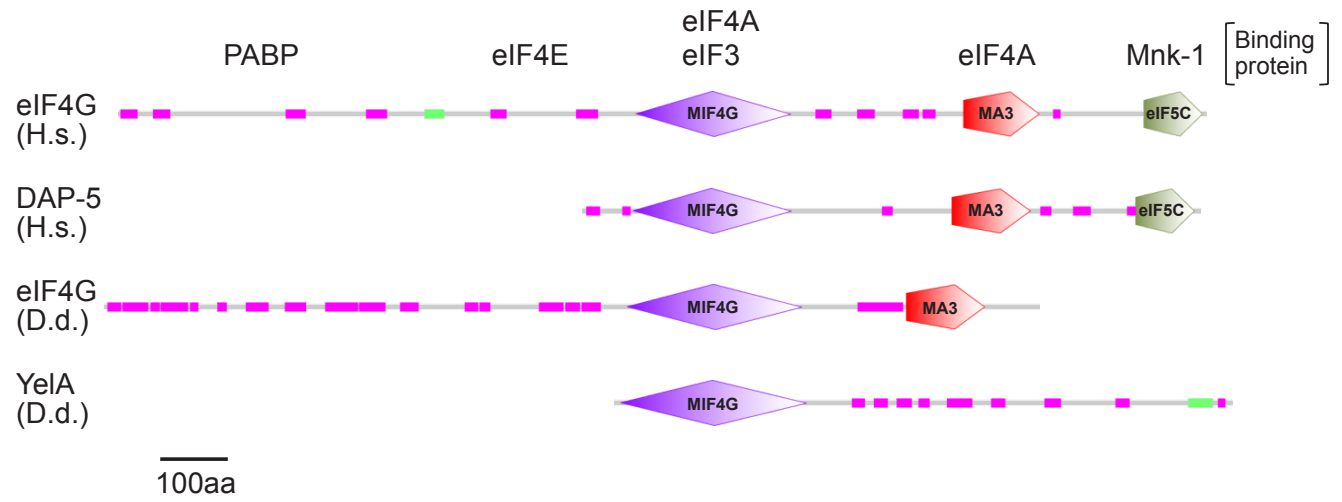

Fig. 1. The domain architecture of human and Dictyostelium MIF4G containing proteins. MIF $4 G$ is shown in purple. Some proteins contain MA3 (red) and elF5C (green) domains. The MA3 domain of Dictyostelium elF4G does not register in SMART, but is identified as a Pfam domain. Low complexity (pink) and coiled-coil (green) regions are shown. Proteins that interact with different part of human elF4G are indicated at the top.

understood for the ecmA-promoter. When multimerised and placed upstream of basal promoter elements, a 22 nucleotide element from within the ecmA promoter directs generic prestalk expression, and is DIF-inducible (Fukuzawa et al., 2006). The element contains an essential binding site for the Myb transcription factor MybE but there is genetic and biochemical evidence for the involvement of three other transcription factors in ecmA gene transcription: the b-Zip proteins, DimA and DimB, and the GATA factor GtaC (Thompson et al., 2004; Huang et al., 2006; Zhukovskaya et al., 2006; Keller et al., 2008) reviewed by (Fukuzawa 2011). DimA, DimB and GtaC accumulate rapidly in the nuclei of cells induced with DIF-1 and DimB contains a phosphorylation site, very near the $C$ terminus, that displays an increased level of modification in DIF-treated cells
(Yamada et al., 2013). The signaling steps upstream from DimB phosphorylation are, however, entirely unknown.

In order to identify potential signaling intermediates, we previously used global proteomic methodology to identify sites that changed phosphorylation level within minutes of exposure to DIF-1 (Sugden et al., 2015). The results identified many proteins that undergo a transient net dephosphorylation at specific sites. The gene encoding one of these, yelA, was originally discovered in an insertional mutagenesis screen and was found to play an essential role in terminal differentiation on both the stalk and spore pathways (Osherov et al., 1997). We identify seven DIF-regulated phosphorylation sites in YelA, all of which are dephosphorylated rapidly in response to DIF-1.
A
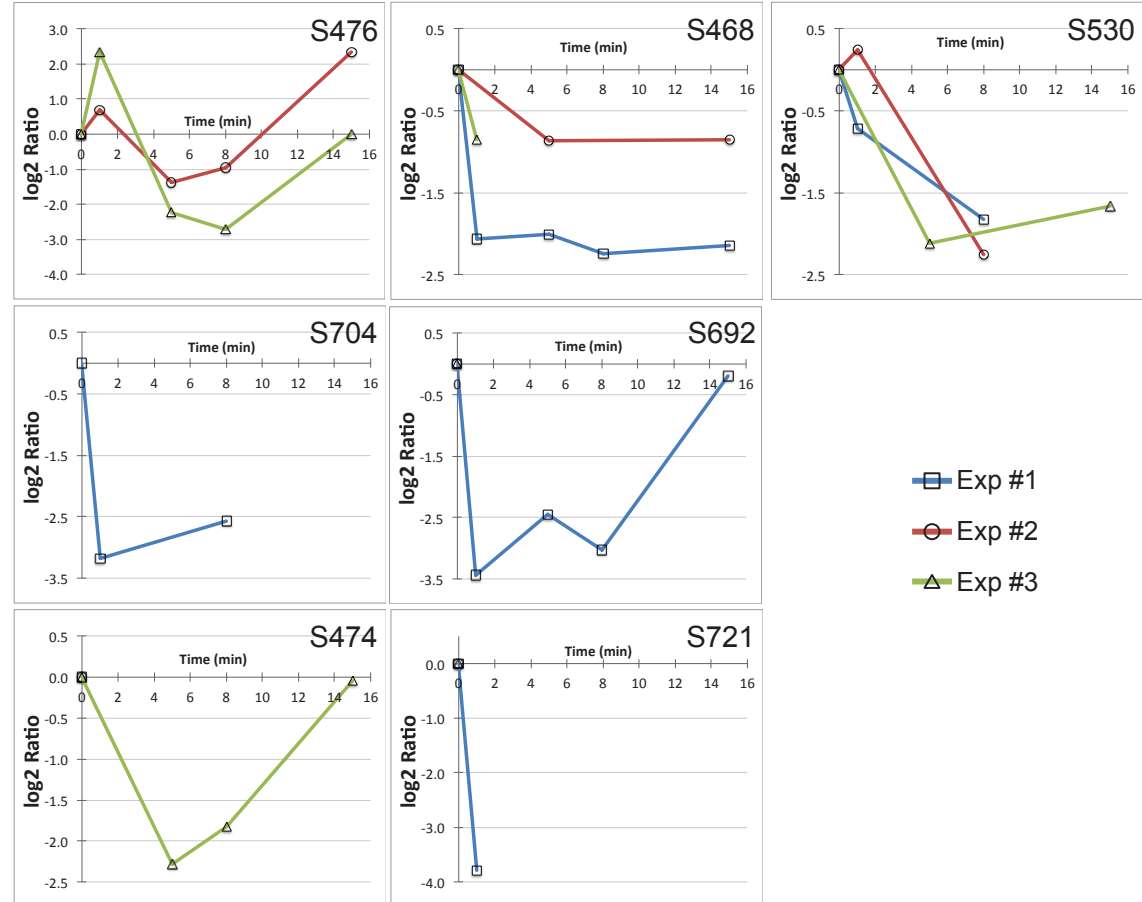

B

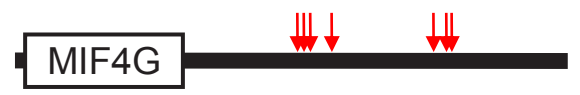

C

7 DIF regulated $\mathrm{P}$-sites

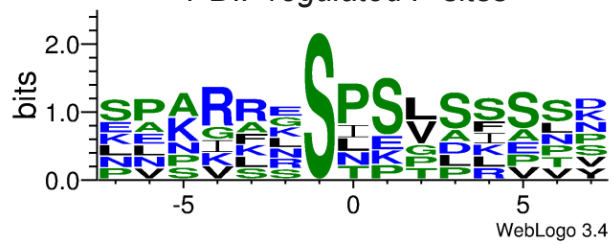

モExp\#1

$-\operatorname{Exp} \# 2$

$\triangle \operatorname{Exp} \# 3$

Fig. 2. Differentiation-inducing factor (DIF)-regulated phosphorylation sites in YelA. (A) Temporal profile of phosphorylation changes in response to DIF-1 stimulation. Level of phosphorylation is expressed as log2 ratio relative to pretreatment. Results from three biological experiments are shown in different colours. (B) Positions of the DIF-regulated phosphorylation sites (red arrows) in the YelA protein. (C) A sequence logo of the seven phosphorylation sites in which amino acids are color coded according to their hydrophobicity: hydrophobic, black; neutral, green; hydrophilic, blue. (D) Amino acid sequences of the phosphorylation sites. The phosphorylated serine residue is shown in red. 
YelA contains an MIF4G domain (Osherov et al., 1997), a sequence initially identified as a sub-domain of elF4G but now known to be present in several other proteins of diverse function (see legend to Fig. 3). elF4G is a scaffold protein that recruits key components of the translational machinery to the mRNA; the MIF4G domain in elF4G includes the binding sites for the elF4A RNA helicase and for elF4E, the 5' methyl G cap-binding protein (Fig. 1). Here we present evidence that YelA has a predicted role in setting the ratio of cap-site dependent to cap-site independent translation. Also, we characterize a deletion mutant of yelA that further implicates it in DIF signaling. This is the first suggestion that, in addition to its role as a regulator of transcription (Williams et al., 1987), DIF-1 functions as a translational regulator. The identity of the regulated $\mathrm{mRNA}(\mathrm{s})$ is unknown but the yelA deletion mutant has an excessively large basal disc and is hyper-sensitive to DIF for gene expression suggesting that YelA target(s) encode negatively acting pathway components.

\section{Results}

\section{Seven sites of serine phosphorylation in YelA become rapidly hypo-phosphorylated in cells exposed to DIF-1}

In our previous study we performed a global quantitative screen for phosphorylation changes that occur within the first few minutes after addition of DIF-1, using a triple-label SILAC approach (Sugden et al., 2015). Several thousand peptides were detected that changed phosphorylation level at least two-fold in response to DIF-1. Only a few of these proteins were characterized further: the two forms of Protein Kinase B, PKBA and PKBR, the MAP kinase Erk2 and calcineurin. Here we characterize one of the other proteins, YelA. We identify seven DIF-regulated sites in YelA and they all follow the most common pattern, whereby DIF-1 causes net dephosphorylation (Fig. 2A-D).

Residues surrounding the phosphorylated serine do not show a strong consensus sequence. However arginine at $P-3$ and serine at $\mathrm{P}+2, \mathrm{P}+4$ and $\mathrm{P}+6$ are common as is proline at $\mathrm{P}+1$ (all numbered relative to the phosphorylated residue) (Fig. 2C, 2D).

\section{YelA has the characteristics of a translational regulator}

YelA contains a MIF4G domain, immediately proximal to its $\mathrm{N}$-terminus and therefore well separated from the sites of DIF-1 inhibitable phosphorylation (Fig. 2B and (Osherov et al., 1997). The MI4FG domain was discovered in the elF4G scaffolding protein and is also present in other components of the translational machinery. It is also, however, present in proteins involved in RNA splicing and in nonsense codon-mediated mRNA decay (see legend to Fig. 3). Dictyostelium encodes orthologues of all these proteins. Fig. 3 panel A is a "SMART" analysis (Letunic et al., 2012) of the seven membered Dictyostelium MIF4G domain family and panel B is the tree that derives from an alignment of the MIF4G domains. The YelA MIF4G domain clusters most closely with that of elF4G itself and with a very close orthologue that we term elF4G-like.

\section{The YelA null strain forms aberrant fruiting bodies in which prestalk and stalk markers are mis-expressed and that have highly enlarged basal discs}

In order to determine whether YelA does indeed have a role in DIF signaling, we generated a deletion strain by homologous recombination, removing most of the MIF4G domain (Fig. 4). The mutant forms aberrant fruiting bodies over approximately the same time course as the parental, Ax2 strain. This is in radical contrast to the published phenotype (Osherov et al., 1997). YelA was identified in a REMI screen for developmental mutants. Both the original REMI mutant and a freshly generated insertion mutant, with a more cap site proximal insertion point, arrest development when cells form mound shaped aggregates prior to slug formation. These mounds contain environmental insult-sensitive cells that accumulate yellow pigment. There are two straightforward explana-

\section{A}

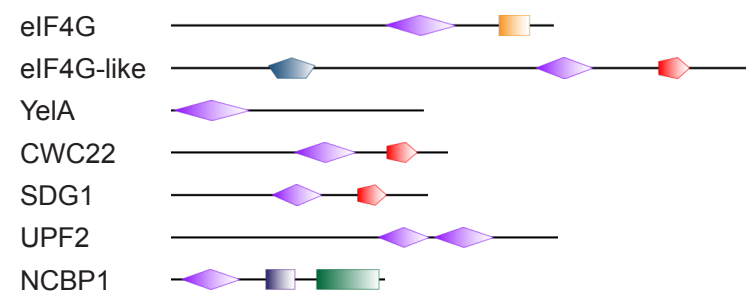

$\begin{array}{|ll|}\square & \text { MIF4G } \\ \square & \text { MIF4G_like, type1 } \\ \text { MIF4G_like, type2 } \\ \text { MA3 } \\ \text { MA3 (Pfam) } \\ \text { VWA }\end{array}$

B

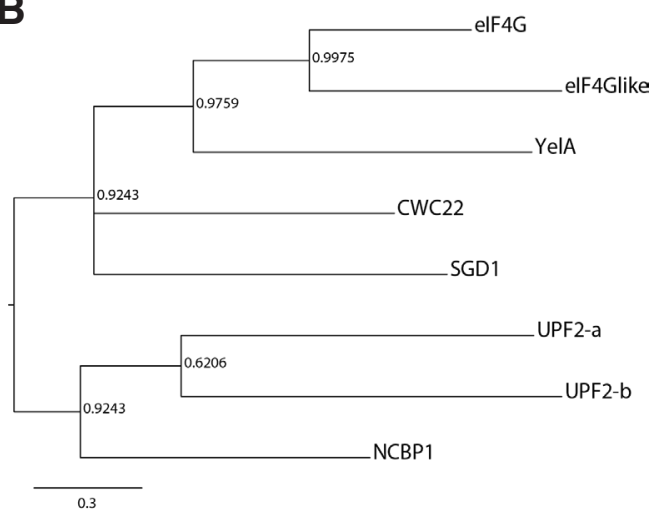

Fig. 3. D. discoideum MIF4G containing proteins. The identities of the proteins were assigned by machine annotation at dictyBase. (A) The domain architectures of the proteins were analysed using SMART. Domains are shown with different colours as indicated in the panel. Regions of low complexity and coiled coil are not shown for simplicity. (B) The sequences of the MIF4G domains were aligned using COBALT and phylogeny was constructed by Bayesian inference. Numbers at nodes indicate probability. The two MIF4G domains of Ufp-2 are shown as Ufp2-a and b. elF4G; DDB_G0275395, is the protein designated elF4G at dictyBase. elF4G-like; DDB_G0286969. CWC22; DDB_G0267796, is a pre-mRNA splicing factor that is associated with the spliceosome. SGD1 (NOM1); DDB_G0284539 is a nucleolar protein that is required for $18 S$ rRNA biogenesis. NCBP1 (CBP80); DDB_G0269814, Nuclear cap binding protein 1 is responsible for a "pioneer" round of translation designed to detect defective mRNAs. Upf2; DDB_G0281623, is a nuclear protein that mediates the decay of mRNAs encoding a premature stop codon. 


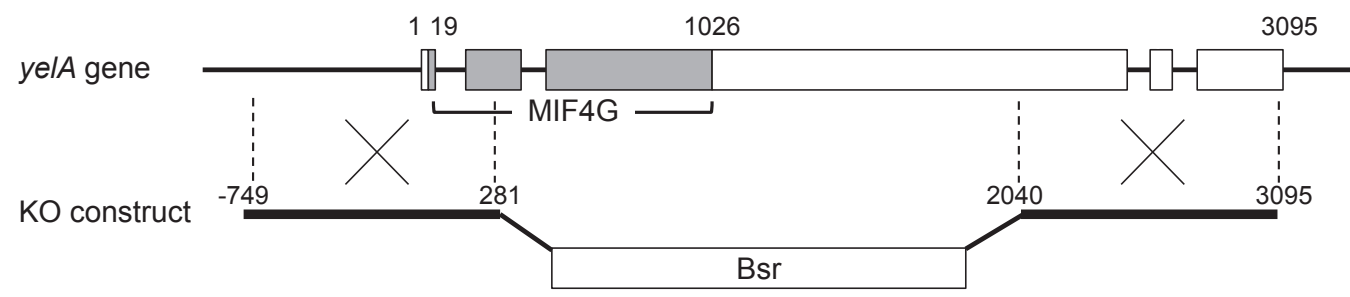

Fig. 4. Disruption of the yelA gene. The genomic structures of yelA and the yelA knockout (KO) construct are shown. Bsr; blasticidin resistant cassette. The MIF4G domain (grey) encompasses nucleotides 19 to 1026

tions for this disparity. It could reflect the fact that different parental strains were used: AX4 in the original study and Ax2 here. There are other examples where disruption of the same gene produces a more severe phenotype in AX4 (Nelson et al., 2000; Schilde et al., 2004). Alternatively, it may result from differences in the genetic lesion; the original REMI mutant and the re-disrupted mutant were single site insertions while the new mutant is a deletion mutant that removes a significant part of the gene including most of the MIF4G domain (Fig. 4). An important feature of the latter explanation is

A

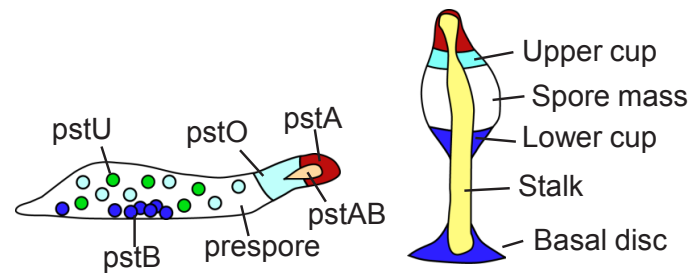

B

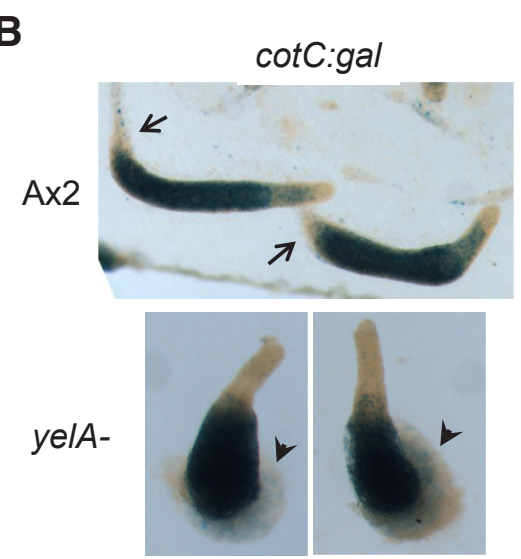

D

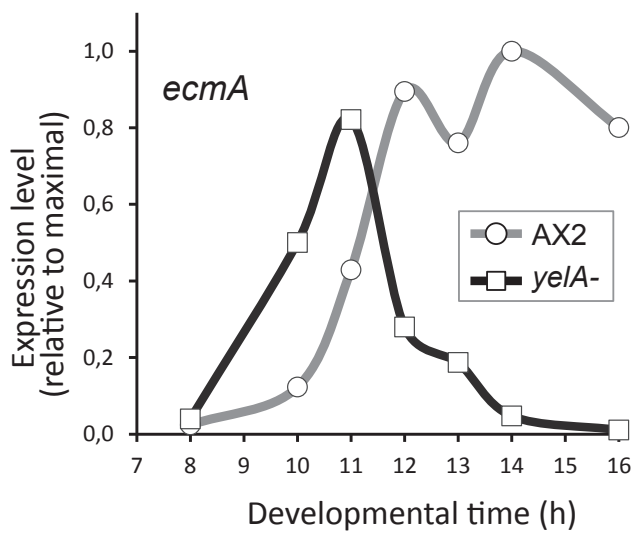

the implication that a more extensive mutation gives a weaker phenotype. This could most simply be explained if the previously described insertional mutants display a dominant negative effect that is not shown by the deletion mutant.

As mentioned, the series of morphological changes that shape the fruiting body, such as tip formation at the apex of the mound, occur at approximately the same times in the YelA null ( $y e l A-)$ strain as in the parent. However, the final fruiting body is aberrant. The spore head fails to ascend the stalk, there is often a clearly demarcated band of cells near the apex and the basal disc is greatly over-sized. These features can best be visualized and understood using cell-type specific markers.

CotC is a protein of the spore coat and the gene is selectively expressed in prespore cells. In slugs or first fingers expressing cotC:gal, there is an unstained region at the posterior that will at culmination contribute to the outer part of the basal disc. This region is greatly expanded in the yelA-strain (Fig. 5B). We also have positive staining data, confirming our designation of the pre-basal disc cells and identifying the apical band of cells. This derives from analyse of ecmA and ecmBexpression. The ecmA:gal marker is, in parental structures, selectively expressed in pstA and pstO cells. They populate the front one-fifth of the slug and also constitute scattered cells within the prespore region: the ALC. It is also expressed in the prebasal disc region; weakly, but strongly enough to delineate the structure. In agreement with the $\cot C$ data the stained region is much larger in the yelA- strain (Fig. 5C). This was confirmed using the pstB and pstAB marker, ecmB:gal.

In parental $A \times 2$ cells at the first finger stage ecmB:gal is expressed in ALC and selectively in pstB cells at the base. Once a migratory slug is formed, the pstB population disappears, presumably either deposited onto the substratum or dispersed within the slug, and a cone of ecmB-expressing cells appears in the tip. Because these cells also express ecmA they are called pstAB cells. At very long

Fig. 5. Expression of prespore and prestalk marker genes in Ax2 and yelA- cells. (A) Schematic diagram of cell type patterning in a slug and a culminating structure. The stalk derives from prestalk cells that occupy the anterior region of the slug: pstA cells and pstO cells. Within the anterior region is a core of pstAB cells that will form the inner part of the basal disc. The outer region of the basal disc is formed from pstB cells, which reside at the rear, substrate proximal region of the slug. Some of the pstB cells also form a lower cup that supports the spore cell mass, whereas some of the pstO cells and another sub-type, the pstU cells form an upper cup above the spore mass (Yamada et al., 2010). (B, C) Ax2 and yelAcells expressing cotC:gal (B) or ecmA:gal (C) were developed for $14.5 \mathrm{~h}$, fixed and stained for $\beta$-galactosidase. Prebasal disc region at the rear of Ax2 slug (arrow) and the corresponding region in yelA- (arrow head) are indicated. (D) RNA was prepared from Ax2 (circle) or yelA- (square) cells at the indicated times of development and analysed for ecmA expression by $R T-q P C R$. Expression is shown relative to the maximal expression observed, that of Ax2 cells at $14.5 \mathrm{~h}$. 
times of staining like that shown in Fig. 6A, there is apparent weak expression in the region of the pstO zone. Staining in this position increases dramatically at culmination to form a structure called the upper cup and there is most often a lower cup, below the spore head. The inner part of the basal disc is formed by the downward movement of the stalk, which embeds itself into the cone of prebasal disc cells that form the outer basal disc. In the yelA-strain there is a much higher apparent level of ecmB:gal expression and it is highly precocious. Staining occurs in minutes rather than hours and there is considerable, apparently ectopic, expression. The upper cup is particularly prominent and premature; it stains strongly during the finger stage and through into culmination. Again, as is visualized with the $\cot C$ and $e c m A$-derived markers, the prebasal disc and outer basal disc are expanded.

The above effects on cellular differentiation, although pronounced, are quantitative rather than qualitative. We therefore analysed a less widely expressed ecmB promoter-derived marker, ST:gal (Ceccarelli et al., 1991). This is normally expressed only within the stalk tube cells but in the yelA-strain it is also expressed in the outer part of the basal disc (Fig. 6B). Thus there is qualitative ectopic gene expression in the mutant.

\section{ecm A and ecmB are prematurely expressed in the yelA- strain}

Staining of the ecmA:gal and ecmB:gal expressing structures gives a rough guide to the relative timing and extent of expression in the parental vs the yelA-strain. However, analysis of the endogenous ecm $A$ and $e c m B$ transcripts can be made truly quantitative. Thus RT-qPCR was performed on parental and yelA-RNAs isolated every two hours from $8 \mathrm{hr}$ to $16 \mathrm{hr}$ of development (Fig. 5D and Fig. $6 \mathrm{C})$. In the case of ecmA the rise in mRNA abundance occurs just one or two hours earlier in the yelA-strain, the peak is achieved earlier and the decline is much more rapid. In the case of ecmB there is a much more radical difference. A barely detectable rise in abundance in parental cells does not occur until $14 \mathrm{hr}$ while in the yelA-strain the mRNA is at $50 \%$ of its peak value by $10 \mathrm{~h}$. When the staining times are taking into consideration, these PCR data seem to be in accord with the marker expression patterns.
A

Ax2
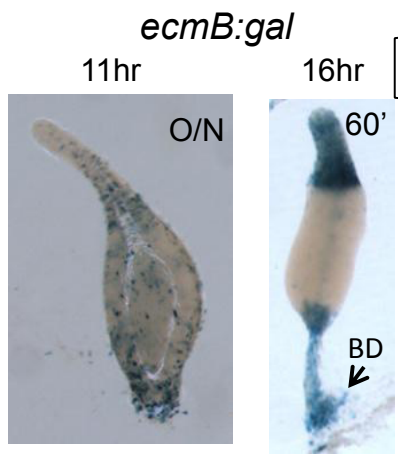

yelA-

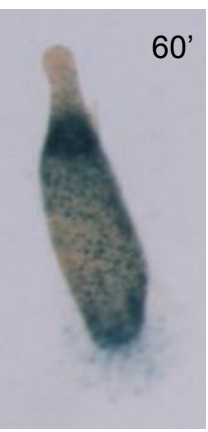
$16 \mathrm{hr}\left[\begin{array}{l}\text { Time of } \\ \text { development }\end{array}\right]$
B

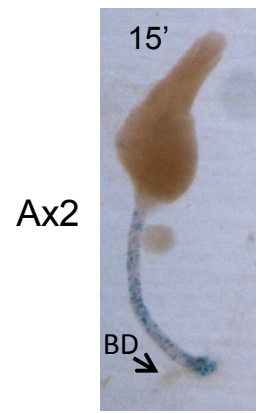

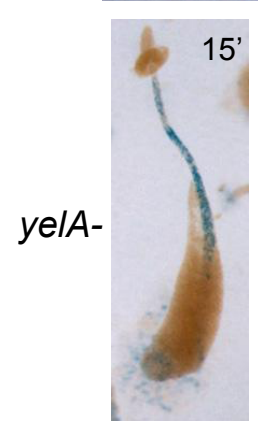

ST:gal

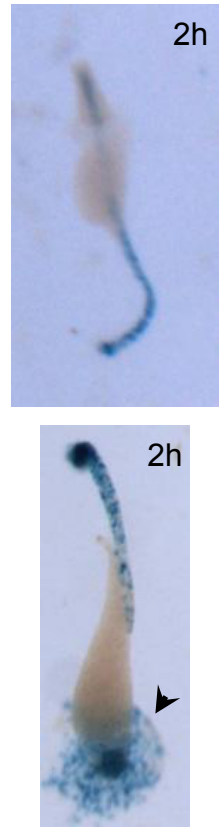

C

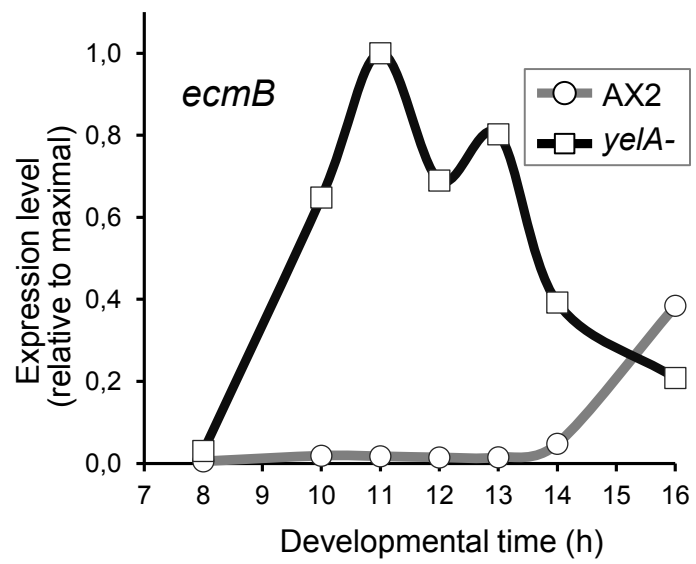

ecmA and ecmB are hyper-inducible by DIF-1 in the yelA- strain

The ecmA and ecmB genes are both inducible by DIF-1 in a monolayer assay. Given the effects of the yelA null mutation on gene expression and gross anatomy, it was of interest to compare sensitivity to DIF-1. In the assay cells were rendered competent to respond to DIF by starvation as a monolayer, then exposed to vehicle (ethanol) alone or to DIF-1 at 30nM or at 100nM. In Fig. 7A the qPCR results for ecmA and ecmB are in each case normalized to the maximum value obtained; i.e. when the yelA- strain is induced at $100 \mathrm{nM}$. This reveals a major difference between the parental and the yelA null, with the mutant showing gross overexpression of both markers. The mutant is not however entirely DIF insensitive. This becomes apparent when the same data set is normalized to the value at $100 \mathrm{nM}$ DIF-1 for each strain separately (Fig. 7B). As the concentration of DIF-1 rises from zero the level of expression also rises in the mutant strain.

Fig. 6. Expression of the ecmB gene in Ax2 and yelA-cells. $A \times 2$ and yelA-cells transformed with ecmB:gal (A) or a stalk tube specific ST:gal (B) were developed, fixed and stained for $\beta$-galactosidase. In (A), cells were developed for indicated times and the 11 hour-structure of Ax2 was stained overnight $(O / N)$, while other structures were stained for $60 \mathrm{~min}$. In (B), 17 hour-developed structures were used. The staining times are indicated on each panel. In both $(A, B)$, basal disc of culminating structures of $A \times 2$ (arrow) and the corresponding region ofyelA- (arrow head) are indicated. (C) RNA was prepared from Ax2 (circle) or yelA- (square) cells at the indicated times of development and analysed for ecmB expression by RT-qPCR. Expression is shown relative to the maximal expression (yelA at $11 \mathrm{~h}$ ). 


\section{Discussion}

The seven sites where DIF-1 treatment exerts an effect on YelA phosphorylation

It is important to know whether the same kinase modifies YelA at the seven regulated sites. Although there is no strong consensus sequence surrounding the phosphorylated serine residue, there are some common features such as arginine at $P-3$, serine at $\mathrm{P}+2, \mathrm{P}+4$ and $\mathrm{P}+6$ and proline at $\mathrm{P}+1$ (Fig. 2C, D). Proline at $\mathrm{P}+1$ was also identified as a prominent residue in many other of the DIF-1 regulated phosphorylation sites (Sugden et al., 2015). This is often indicative of proline-directed kinase regulation such as extracellular-regulated kinase-2 (ERK2) and cyclin-dependent kinases (Sugden etal., 2015). Dephosphorylation levels of the seven sites vary in their maximum response but it is impossible to give precise kinetics for all seven, because the sampling is by its nature highly stochastic. However, the responses were all rapid, transient and the largest decreases were in the order of 10-fold (Fig. 2A).

\section{Insights into the possible function of YelA}

We have performed a bio-informatic analysis of the Dictyostelium MIF4G family of proteins. Based upon MIF4G domain sequence alignments it would appear that YelAis most closely related to elF4G itself and also to a related protein (termed here the "elF4G-like" protein) that contains a von Willebrand Factor A domain ("VWA" in Fig. 3A). The latter fact is not however particularly informative because proteins containing a VWA domain are involved in a very wide range of processes. There is one glaring difference between the domain organization of elF4G and YelA. The N-terminus proximal region of elF4G contains the binding sites for elF4E, the 5' cap binding protein, and PABP, the polyA-binding protein. However, the equivalent region is almost entirely absent from YelA; the MIF4G domain initiates a mere six amino acids from the initiation codon. Such an organization mirrors that of DAP5/P97/NAT1; a metazoan translational regulator that resembles elF4G through most of its length but lacks sequences homologous to the approximate $N$ terminal one third of elF4G. The lack of an elF4E association domain means DAP5 cannot recruit mRNAs by binding to their caps, but retains the ability to initiate cap-independent translation via any IRES elements contained within the mRNA (Lee et al., 2006; Lewis et al., 2008; Liberman et al., 2008; Weingarten-Gabbay et al., 2014; Liberman et al., 2015).

\section{YelA is only a partial orthologue of DAP5}

Although there is this striking similarity of architecture and detailed sequence of the MIF4G domain, YelA is not a complete orthologue of DAP5. Metazoan DAP5 and elF4G both possess a $\mathrm{C}$ terminal domain that binds the Mnk-1 kinase. This is absent from both Dictyostelium YelAand elF4G. YelAalso lacks an MA3 domain, downstream of the MIF4G domain, such as is frequently found in MIF4G containing proteins including DAP5 (Fig. 3A). However this may be misleading because the MA3 domain in DAP5 does not bind elF4A (Imataka et al., 1997). Thus there are potentially significant differences between YelA and DAP5. However, we strengthen the case that YelA is the closest orthologue of DAP5 by showing that, although there are direct equivalents of most of

A
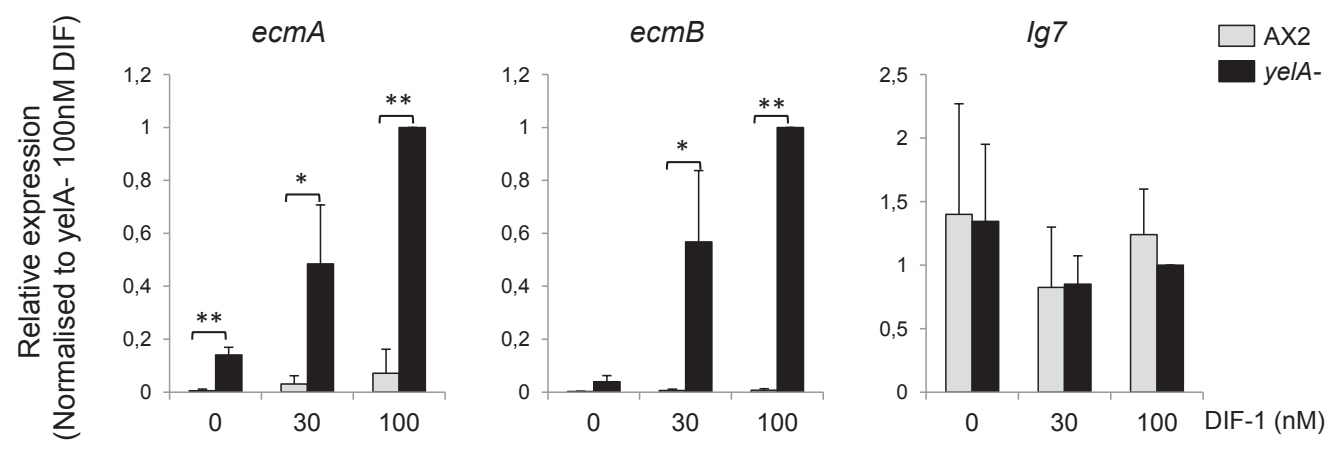

B
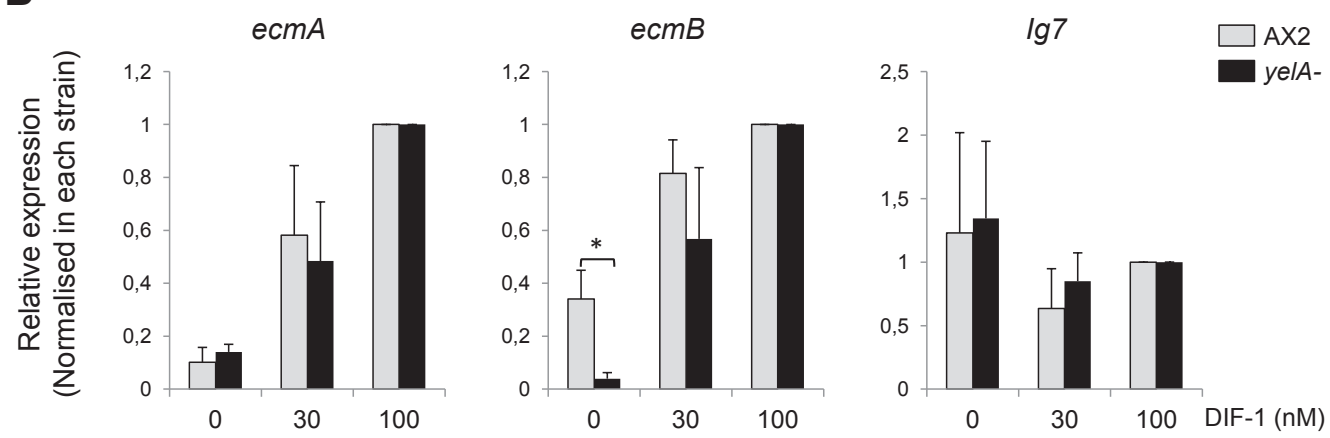

Fig. 7. Differentiation-inducing factor (DIF) sensitivity of prestalk gene induction in $\mathbf{A x 2}$ and yelA- cells. Ax2 and yelA- cells were incubated in monolayer with different concentration of DIF-1. After 2 hours, RNA was extracted and analysed for gene expression by RT-qPCR. (A) Expression is normalised to expression in yelA- cells treated with 100nM DIF-1. (B) The same result is presented as a fraction of expression with $100 \mathrm{nM}$ DIF-1 within

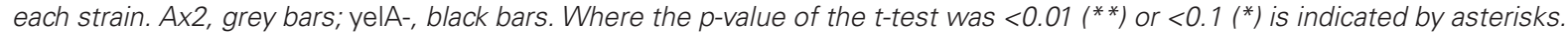


the MIF4G containing proteins, there are, within the sequences of the MIF4G domains, no closer matches to DAP5. Thus, by a process of exclusion, we can be confident that there are no better candidates for the role of DAP5.

\section{How does YelA regulate DIF-1 signaling?}

The necessity to utilize IRES elements, rather than the cap site, confers potential selectivity on the translational process and DAP5 is believed to act in this way to facilitate translation of cell cycle regulated and stress regulated mRNAs (Lewis et al., 2008; Marash et al., 2008; Liberman et al., 2009). These include Bcl-2, Cdk1 and p53. In the case of YelA, its domain structure suggests strongly that it is a translational regulator that recognizes IRES's in one or more unknown mRNAs encoding components of a DIF signaling pathway. Also, there is the implication that DIF-1 controls this activity via one or more of the phosphorylation events.

\section{YelA as a repressor of DIF-1 signalling}

The increased sensitivity to DIF-1 in the yelA null inversely correlates with the effects of genetically inactivating components of the DIF-synthesis or response machinery; the latter mutations result in reduced basal disc formation while the yelA null mutation results in increased basal disc differentiation. Precocious expression is more prominent with ecmB, a gene that is highly expressed in pstB cells. Therefore yelA functions to supress pstB cell differentiation. DIF-1 seems to antagonize this effect in the precursors of pstB cells, presumably by controling the phosphorylation of YelA. Analysis of a marker that is normally only expressed in the stalk tube confirms that expression is truly ectopic in the yelA-mutant. We do not know the target of the proposed translational control or how it impinges on the DIF signaling pathway. The most telling observation is the increased sensitivity to DIF but we do not know the identity of any other of the direct signaling intermediates, aside from DimB where there is ChIP data for promoter recruitment in response to DIF-1 (Zhukovskaya et al., 2006; Yamada et al., 2013).

\section{Materials and Methods}

\section{Cell culture and development}

Axenically grown cells, strain Ax2 Gerisch isolate, were used. Development, transformation and staining for lacZ expression is done as before (Fukuzawa et al., 2006).

\section{Construction of the yelA knockout plasmid}

For the yelA gene knockout, the yelA gene was amplified with primers GAATTCAGCCAATCAGATCCCTTAAACG and CTCGAGTTACAAAACAAATACATCAGACCCTAAATC and cloned into the pJet1.2 blunt cloning vector (Thermo Scientific, Ltd.). Region from 5' end to EcoRV site at nucleotide 2040 was replaced with a fragment from nucleotide -749 to 281 that was amplified with primers GAATTCGTCTGTATATTTGTCTATTTGGTTGGC and GATATCGATGATGGTTCCTCAACCACC, creating EcoRV site at the junction. The Smal fragment of the blasticidin-resistant cassette from pLPBLP (Faix et al., 2004) was inserted into the EcoRV site.

\section{Gene expression}

RNA was prepared from cells and analysed by RT-qPCR as described previously (Sugden et al., 2011). Primers used were as follows. ecmA; CCGTAAACTGTGAATGTGATGACC and GTCTTGGAATCGCAACTATCAGC, ecmB; CTCTTGATTCATGTTGTTCAACTG and CATCGCCACATTTTCCAAATG, Ig7; TTACATTTATTAGACCCGAAACCAAGCG and AACAGCTATCACCAAGCTTGATTAGCC.

\section{DIF sensitivity assay}

$2 \times 10^{6}$ cells were plated in $2.5 \mathrm{ml}$ of stalk buffer $(10 \mathrm{mM}$ MES-KOH pH $6.2,10 \mathrm{mM} \mathrm{KCl}, 2 \mathrm{mM} \mathrm{NaCl}, 1 \mathrm{mM} \mathrm{CaCl}_{2}$ ) in a $6 \mathrm{~cm}$ dish and exposed to $4 \mathrm{mM}$ cAMP in fresh stalk buffer containing $50 \mu \mathrm{M}$ cerulenin for $1 \mathrm{~h}$. After incubating for 7 hours to attain competence to respond to DIF, cells were induced in fresh stalk buffer containing $50 \mu \mathrm{M}$ cerulenin and different concentrations of DIF-1. After 2 hours RNA was prepared and gene expression was analysed by RT-qPCR.

\section{Protein domain structure and phylogeny analysis}

Protein domain architecture was analysed with SMART (Letunic et al., 2012). Sequence of MIF4G domains (SM00543) is extracted and aligned using COBALT (Papadopoulos et al., 2007). Phylogeny is constructed by Bayesian inference using a mixed amino acid model with rate variation between sites estimated by a gamma distribution. Analysis was run for $4 \times 10^{5}$ generations.

\section{Acknowledgements}

This work was initially supported by Wellcome Trust Program Grant 053640/Z to J.G.W and completed while he was in receipt of Leverhulme Emeritus Fellowship EM-2013-024.

\section{References}

CECCARELLI A, MAHBUBANI H, WILLIAMS J G (1991). Positively and negatively acting signals regulating stalk cell and anterior-like cell differentiation in Dictyostelium. Cell 65: 983-989.

FAIX J, KREPPEL L, SHAULSKY G, SCHLEICHER M, KIMMEL A R (2004). A rapid and efficient method to generate multiple gene disruptions in Dictyostelium discoideum using a single selectable marker and the Cre-loxP system. Nucleic Acids Res 32: e143.

FUKUZAWA M (2011). Control of prestalk-cell differentiation by transcription factors. Dev Growth Differ 53: 538-547.

FUKUZAWA M, ZHUKOVSKAYA N V, YAMADA Y, ARAKI T, WILLIAMS J G (2006). Regulation of Dictyostelium prestalk-specific gene expression by a SHAQKY family MYB transcription factor. Development 133: 1715-1724.

GAUDET P, WILLIAMS J G, FEY P, CHISHOLM R L (2008). An anatomy ontology to represent biological knowledge in Dictyostelium discoideum. BMC Genomics 9: 130.

HUANG E Y, BLAGG S L, KELLER T, KATOH M, SHAULSKY G, THOMPSON C R $L$ (2006). bZIP transcription factor interactions regulate DIF responses in Dictyostelium. Development 133: 449-458.

IMATAKA H, SONENBERG N (1997). Human eukaryotic translation initiation factor 4G (elF4G) possesses two separate and independent binding sites for elF4A. Mol Cell Biol 17: 6940-6947.

JERMYN K A, DUFFY K T, WILLIAMS J G (1989). A new anatomy of the prestalk zone in Dictyostelium. Nature 340: 144-146.

KAY R R, FLATMAN P, THOMPSON C RL (1999). DIF signalling and cell fate. Semin. Cell Dev. Biol. 10: 577-585.

KELLER T, THOMPSON C R (2008). Cell type specificity of a diffusible inducer is determined by a GATA family transcription factor. Development 135: 1635-1645.

LEE S H, MCCORMICK F (2006). p97/DAP5 is a ribosome-associated factor that facilitates protein synthesis and cell proliferation by modulating the synthesis of cell cycle proteins. EMBO J. 25: 4008-4019.

LETUNIC I, DOERKS T, BORK P (2012). SMART 7: recent updates to the protein domain annotation resource. Nucleic Acids Res 40: D302-305.

LEWIS S M, CERQUOZZI S, GRABERT E, UNGUREANU N H, ANDREWS M, HOLCIK M (2008). The elF4G homolog DAP5/p97 supports the translation of select mRNAs during endoplasmic reticulum stress. Nucleic Acids Res 36: 168-178.

LIBERMAN N, DYM O, UNGER T, ALBECK S, PELEG Y, JACOBOVITCH Y, BRANZBURG A, EISENSTEIN M, MARASH L, KIMCHI A (2008). The crystal structure of the C-terminal DAP5/p97 domain sheds light on the molecular basis for its processing by caspase cleavage. $\mathrm{J} \mathrm{Mol} \mathrm{Biol} \mathrm{383:} \mathrm{539-548.}$

LIBERMAN N, GANDIN V, SVITKIN Y V, DAVID M, VIRGILI G, JARAMILLO M HOLCIK M, NAGAR B, KIMCHIA, SONENBERG N (2015). DAP5 associates with elF2beta and elF4AI to promote Internal Ribosome Entry Site driven translation. 


\section{Y. Yamada et al.}

Nucleic Acids Res 43: 3764-3775.

LIBERMAN N, MARASH L, KIMCHI A (2009). The translation initiation factor DAP5 is a regulator of cell survival during mitosis. Cell Cycle 8: 204-209.

MARASHL, LIBERMAN N, HENIS-KORENBLITS, SIVANG, REEME, ELROY-STEIN $\mathrm{O}, \mathrm{KIMCHI}$ A (2008). DAP5 promotes cap-independent translation of $\mathrm{Bcl}-2$ and CDK1 to facilitate cell survival during mitosis. Mol Cell 30: 447-459.

MCROBBIE S J, JERMYN K A, DUFFY K, BLIGHT K, WILLIAMS J G (1988). Two DIF-inducible, prestalk-specific mRNAs of Dictyostelium encode extracellular matrix proteins of the slug. Development 104: 275-284.

NELSON M K, CLARK A, ABE T, NOMURAA, YADAVA N, FUNAIR C J, JERMYN K A, MOHANTY S, FIRTEL R A, WILLIAMS J G (2000). An F-Box/WD40 repeatcontaining protein important for Dictyostelium cell-type proportioning, slug behaviour, and culmination. Dev Biol 224: 42-59.

OSHEROV N, WANG N, LOOMIS W F (1997). Precocious sporulation and developmental lethality in yelA null mutants of Dictyostelium. Dev. Genet. 20: 307-319.

PAPADOPOULOS J S, AGARWALA R (2007). COBALT: constraint-based alignment tool for multiple protein sequences. Bioinformatics 23: 1073-1079.

SCHILDE C, ARAKI T, WILLIAMS H, HARWOOD A, WILLIAMS J G (2004). GSK3 is a multifunctional regulator of Dictyostelium development. Development 131: 4555-4565.

SUGDEN C, ROSS S, ANNESLEY S J, COLE C, BLOOMFIELD G, IVENS A SKELTON J, FISHER P R, BARTON G, WILLIAMS J G (2011). A Dictyostelium $\mathrm{SH} 2$ adaptor protein required for correct DIF-1 signaling and pattern formation. Dev Biol 353: 290-301.

SUGDEN C, URBANIAK M D, ARAKI T, WILLIAMS J G (2015). The Dictyostelium prestalk inducer differentiation-inducing factor-1 (DIF-1) triggers unexpectedly complex global phosphorylation changes. Mol Biol Cell 26: 805-820.

THOMPSON C R L, FU Q, BUHAY C, KAY R R, SHAULSKY G (2004). A bZIP/bRLZ transcription factor required for DIF signaling in Dictyostelium. Development 131: 513-523.

THOMPSON C R L, KAYRR (2000). Cell-fate choice in Dictyostelium: intrinsic biases modulate sensitivity to DIF signaling. Dev. Biol. 227: 56-64.

WANG M, VANDRIELR, SCHAAP P (1988). Cyclic AMP-phosphodiesterase induces dedifferentiation of prespore cells in Dictyostelium discoideum slugs: evidence that cyclic AMP is the morphogenetic signal for prespore differentiation. Develop ment 103: 611-618.

WEINGARTEN-GABBAY S, KHAN D, LIBERMAN N, YOFFE Y, BIALIK S, DAS $\mathrm{S}$, OREN M, KIMCHI A (2014). The translation initiation factor DAP5 promotes IRES-driven translation of p53 mRNA. Oncogene 33: 611-618.

WILLIAMS J G, CECCARELLIA, MCROBBIE S, MAHBUBANI H, KAYRR, FARLYA BERKS M, JERMYN K A (1987). Direct induction of Dictyostelium prestalk gene expression by DIF provides evidence that DIF is a morphogen. Cell 49: 185-192.

YAMADA Y, KAY R R, BLOOMFIELD G, ROSS S, IVENS A, WILLIAMS J G (2010). A new Dictyostelium prestalk cell sub-type. Dev Biol 339: 390-397.

YAMADA Y, KUBOHARA Y, KIKUCHI H, OSHIMAY, WANG HY, ROSS S, WILLIAMS J G (2013). The Dictyostelium prestalk inducer DIF-1 directs phosphorylation of a bZIP transcription factor. Int J Dev Biol 57: 375-381.

ZHUKOVSKAYA N V, FUKUZAWA M, YAMADA Y, ARAKI T, WILLIAMS J G (2006) The Dictyostelium bZIP transcription factor DimB regulates prestalk-specific gene expression. Development 133: 439-448. 


\section{Further Related Reading, published previously in the Int. J. Dev. Biol.}

The Dictyostelium prestalk inducer DIF-1 directs phosphorylation of a bZIP transcription factor

Yoko Yamada, Yuzuru Kubohara, Haruhisa Kikuchi, Yoshiteru Oshima, Hong-Yu Wang, Susan Ross and Jeffrey G. Williams Int. J. Dev. Biol. (2013) 57: 375-381

http://dx.doi.org/10.1387/ijdb.130046jw

An orthologue of the Myelin-gene Regulatory Transcription Factor regulates Dictyostelium prestalk differentiation Hiroshi Senoo, Hong-Yu Wang, Tsuyoshi Araki, Jeffrey G. Williams and Masashi Fukuzawa Int. J. Dev. Biol. (2012) 56: 325-334

http://dx.doi.org/10.1387/ijdb.120030jw

ASET/MYND chromatin re-modelling protein regulates Dictyosteliumprespore patterning Beatriz Nuñez-Corcuera, Joanna Birch and Jeffrey G. Williams Int. J. Dev. Biol. (2011) 55: 205-208

http://dx.doi.org/10.1387/ijdb.113309bn

Synergy between two transcription factors directs gene expression in Dictyostelium tiporganiser cells

Hong Yu Wang and Jeffrey G. Williams

Int. J. Dev. Biol. (2010) 54: 1301-1307

http://dx.doi.org/10.1387/ijdb.103141hw

Identification of a target for CudA, the transcription factor which directs formation of the Dictyostelium tip organiser

Hong-Yu Wang and Jeffrey G. Williams

Int. J. Dev. Biol. (2010) 54: 161-165

http://dx.doi.org/10.1387/ijdb.082723hw

5 yr ISI Impact Factor $(2013)=2.879$

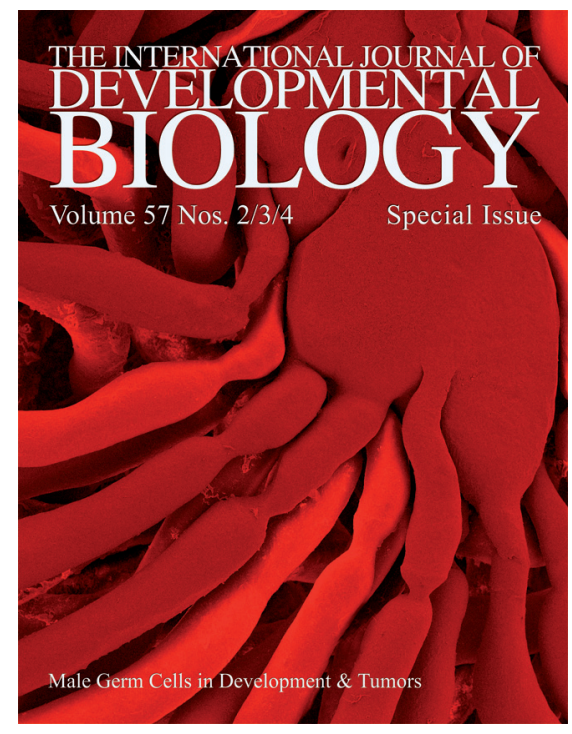

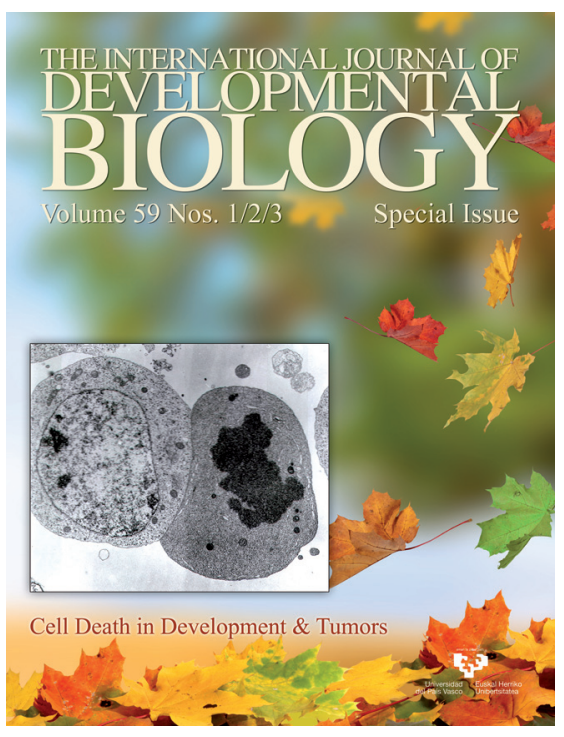
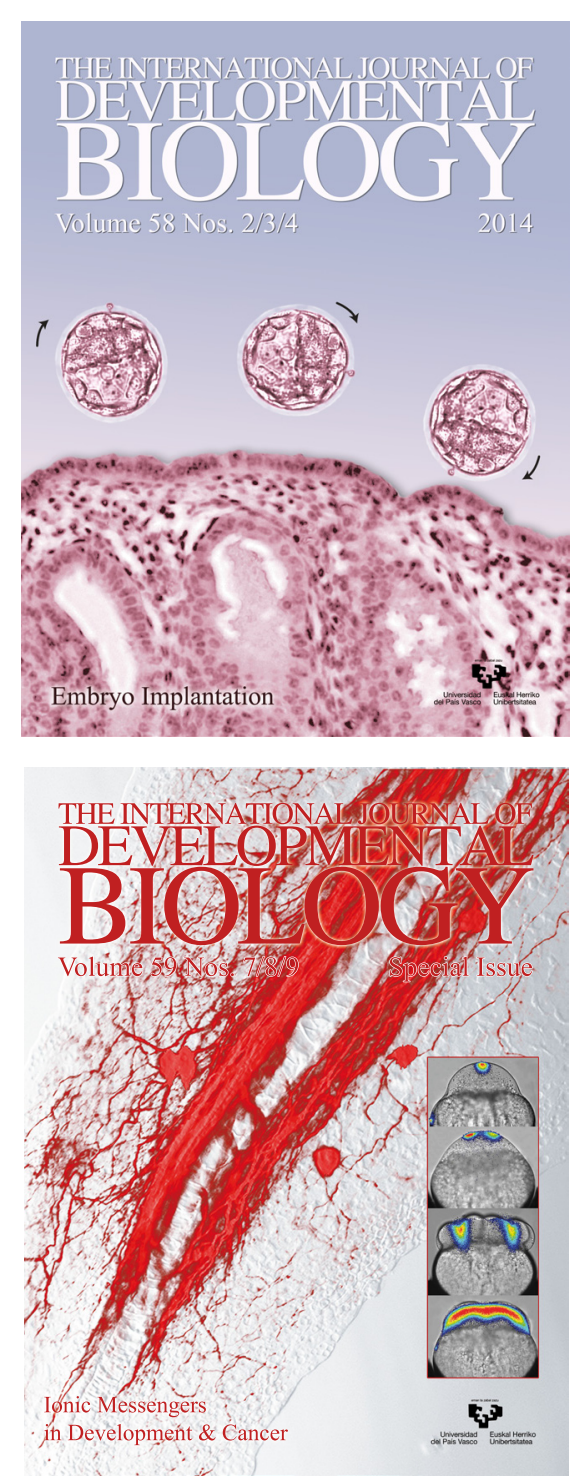